\title{
DEL PORQUÉ LA REGENERACIÓN NATURAL ES TAN IMPORTANTE PARA LA COEXISTENCIA DE ESPECIES EN LOS BOSQUES TROPICALES
}

\author{
On the reasons that natural regeneration is important for species \\ coexistence in tropical forests
}

Palabras clave: bosques tropicales, coexistencia de especies, dispersión de semillas, heterogeneidad ambiental, regeneración.

Key words: tropical forests, seed dispersal, environmental heterogeneity, regeneration.

\section{RESUMEN}

La regeneración natural juega un papel fundamental en el mantenimiento de la diversidad de los bosques tropicales. Dicho proceso ocurre en múltiples fases: producción y dispersión de semillas, germinación y establecimiento de las plántulas. Cada una de estas fases representa un cuello de botella muy fuerte en la demografía de las especies, pues los estadios más tempranos en el ciclo de vida de las plantas (semillas y plántulas) son los más vulnerables a aleas de origen ambiental y biótico, y por ende los individuos están sujetos a altos riesgos de mortalidad. El resultado de esta serie de filtros determinará la distribución espacial de los propágulos, que a su vez refleja la distribución potencial de los árboles. De esta manera, la dispersión de semillas y los procesos ecológicos que determinan el posterior establecimiento de las plántulas juegan un papel esencial en la estructuración de las comunidades de árboles. En la presente revisión, se pasa revista por los cuatro principales procesos ecológicos que gobiernan la fase de regeneración en los bosques tropicales. El primero, la limitación en la dispersión, es el fracaso de las especies en

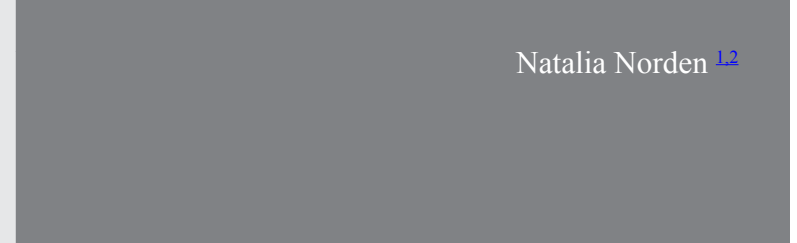

alcanzar un lugar favorable para la regeneración por la ausencia de llegada de semillas. Una vez este obstáculo superado, los factores ambientales constituyen un segundo filtro que puede afectar considerablemente la distribución espacial de las plántulas. Las fluctuaciones temporales en estos procesos generan una variación muy importante en el reclutamiento de plántulas a lo largo del tiempo y le agregan un nuevo componente estocástico a la regeneración. Por último, la abundancia relativa de las especies de plántulas en el sotobosque es regulada por procesos de densidad-dependencia negativa, que limita el reclutamiento de individuos conspecíficos al tiempo que favorece el de individuos de otras especies, mediante el ataque de hongos patógenos y herbívoros.

\begin{abstract}
Plant regeneration plays a critical role in the maintenance of species diversity in tropical rainforests. This is a multistage process, including seed production, dispersal, germination and subsequent seedling establishment. All these stages represent major bottlenecks in plant demography, as early stages in the plant cycle (seeds and seedlings)
\end{abstract}

\footnotetext{
${ }^{1}$ Instituto de Investigación de Recursos Biológicos Alexander von Humboldt, Avenida Paseo Bolívar 16-20, Bogotá D.C., Colombia.

${ }^{2}$ Fundación Cedrela, Diagonal 40A N¹8A-09, Bogotá D.C., Colombia.
} 
are the most vulnerable to environmental hazards, and are therefore subject to high mortality risks. The outcome of these ecological filters will determine not only seedling spatial distribution, but also the potential area of tree distribution. Seed dispersal and subsequent seedling establishment therefore play a critical role in the structuring of tree communities. Here, I review the main four ecological processes driving seedling recruitment in tropical forests. First, dispersal limitation is the failure of seeds to reach suitable microsites for seedling establishment. Once this filter is overcome, environmental factors can considerably affect seedling spatial distribution. Temporal fluctuations in these processes result in an important variation in recruitment success over time, and add a stochastic component to seedling regeneration. Finally, negative-density dependence regulates species relative abundance in the seedling layer by limiting conspecific recruitment through the attack of pathogen, fungi and herbivores.

\section{INTRODUCCIÓN}

Una de las grandes incógnitas de la ecología tropical es cómo miles de especies de árboles coexisten en los bosques tropicales. Estos ecosistemas, que no representan sino el $7 \%$ de las tierras continentales del planeta, resguardan más de la mitad de las especies de Angiospermas descritas (Prance, 1977; Prance et al., 2000). Solamente en Amazonía, se estima que son 16000 las especies de árboles, de las cuales 11000 son consideradas especies raras, pues solo representan el $0.12 \%$ de los individuos (Ter Steege et al., 2013). Esta inmensa diversidad de especies es, en parte, el resultado de diferentes gradientes climáticos y topográficos que van de la mano de cambios en la fertilidad del suelo (Ter Steege et al., 2006). Sin embargo, a escalas más locales, la heterogeneidad ambiental no es tan marcada y todavía es asombroso encontrar hasta 300 especies de árboles en una sola hectárea de bosque Amazónico (Pitman et al., 2001, 2002; Valencia et al., 2004); mientras que en los países templados encontramos en promedio 30 especies de árboles en dichas circunstancias (Whittaker et al., 1956; Masaki et al., 1999).

Reconocer cuáles son los mecanismos que permiten el mantenimiento de la diversidad en los bosques tropicales es un reto que se debe superar si se quieren desarrollar planes de manejo y estrategias de conservación efectivas en los ecosistemas tropicales. En las últimas décadas, los paisajes tropicales han sido rápidamente transformados en un mosaico de cultivos, pastizales y fragmentos de bosque de distintos tamaños, generando una dinámica en la cobertura que ha tenido graves consecuencias para la biodiversidad, el clima y las funciones ecosistémicas a pequeña y gran escala (Lambin et al., 2006; Uriarte et al., 2009). Esta amenaza ha llevado a varios ecólogos y conservacionistas a advertir una posible crisis ambiental causada por la extinción masiva de especies y la pérdida de servicios ambientales, fundamentales para la regulación del clima (Laurance et al., 2007; Hubbell et al., 2008). Por ejemplo, los bosques tropicales son responsables de más de un tercio de la fotosíntesis global de los ecosistemas terrestres (Mellilo et al., 1993) y almacenan aproximadamente el $40 \%$ del carbono que reside en la vegetación (Lewis et al., 2004). Lo anterior convierte a los bosques tropicales en el más importante sumidero de carbono en el mundo. En este contexto, entender cómo se regeneran los bosques tropicales después de haber sido perturbados es fundamental para evaluar su resiliencia en distintos contextos históricos y ecológicos.

La regeneración de los bosques constituye la base para la renovación y la continuidad de las especies, lo que la convierte en uno de los procesos más importantes en el ciclo de vida de las plantas (Nathan \& Muller-Landau, 2000; Wang \& Smith, 2002). Este proceso ocurre en múltiples fases: producción y dispersión de semillas, germinación y establecimiento de las plántulas. Cada una de estas fases representa un filtro ecológico muy importante, pues los estadios más tempranos son los más vulnerables a aleas de origen ambiental y biótico y, por ende, los individuos están sujetos a altos riesgos de mortalidad (Harms et al., 2000; Muller-Landau et al., 2002). El resultado final de esta serie de filtros determinará la distribución espacial de los propágulos, que a su vez refleja la distribución potencial de los árboles. De esta manera, la dispersión de semillas y los 
procesos ecológicos que determinan el posterior establecimiento de las plántulas juegan un papel esencial en la estructuración de las comunidades. Así pues, tener una comprensión global sobre las circunstancias que determinan el reclutamiento exitoso de las especies es un factor crítico para inferir los mecanismos que contribuyen a la continuidad de los bosques tropicales y al mantenimiento de la diversidad en estos ecosistemas (Hubbell et al., 1999; Muller-Landau et al., 2002).

A continuación se pasará revista por los cuatro principales procesos ecológicos que gobiernan la fase de regeneración en los bosques tropicales. Cada uno de ellos representa un cuello de botella que puede limitar el reclutamiento de plantas en el sotobosque. El primero, la limitación en la dispersión, es el fracaso de las especies en alcanzar un lugar favorable para la regeneración por la ausencia de llegada de semillas. Una vez este obstáculo es superado, los factores ambientales constituyen un segundo filtro, que puede afectar considerablemente la distribución espacial de las plántulas. En tercer lugar, las fluctuaciones temporales en estos procesos generan una variación muy importante en el reclutamiento de plántulas a lo largo del tiempo y le agregan un nuevo componente estocástico a la regeneración. Por último, la abundancia relativa de las especies de plántulas en el sotobosque es regulada por procesos de densidad-dependencia negativa, que limita el reclutamiento de individuos conspecíficos al tiempo que favorece el de individuos de otras especies, mediante el ataque de hongos patógenos y herbívoros.

\section{LIMITACIÓN EN LA DISPERSIÓN}

De manera general, el fracaso de las especies por alcanzar un lugar favorable para la regeneración puede ser el resultado de una limitación por ausencia de llegada de semillas (Figura 1a), y/o de una limitación en el establecimiento de las plántulas en el sotobosque (Figura 1b). Varios estudios teóricos han mostrado que la limitación en el reclutamiento por ausencia de llegada de semillas, o limitación en la dispersión, juega un papel fundamental en el mantenimiento de la diversidad (Tilman, 1999; Hurtt \& Pacala, 1995). Si las especies están limitadas en su capacidad de dispersión, las semillas caerán mayoritariamente alrededor de los árboles parentales y los individuos estarán rodeados por conspecíficos con más frecuencia que por heteroespecíficos. Como resultado, la competencia intra-específica será más fuerte que la hetero-específica (Hurtt \& Pacala, 1995; Nathan \& Muller-Landau, 2000), lo que disminuye la exclusión competitiva y promueve la coexistencia. Desde un punto de vista empírico también se ha demostrado que la mayoría de las poblaciones de árboles tropicales están limitadas en su dispersión; por ejemplo, en varias especies de árboles menos de la mitad de las semillas producidas son activamente dispersadas lejos de los parentales (Howe \& Vande Kerckhove, 1981; Howe \& Smallwood, 1982; Ratiarison, 2003). Además, la depredación de las semillas es una de las mayores fuentes de mortalidad a lo largo del ciclo de vida de las plantas. En algunos casos, más del $75 \%$ de las semillas, después de la dispersión, no llega a germinar (Howe et al., 1985; Schupp, 1988) y la mortalidad puede alcanzar hasta el $100 \%$, incluso antes de que las semillas sean dispersadas (Janzen, 1969). Estos filtros ecológicos hacen que las especies fracasen en la colonización de nuevos micrositios potencialmente favorables a su regeneración y que el reclutamiento quede confinado principalmente cerca a los árboles parentales (ubbell, 1980).

Por otra parte, la dispersión de semillas también contribuye a la coexistencia de las especies a través de algunos compromisos evolutivos, o tradeoff. Por ejemplo, el trade-off entre la capacidad de dispersión de las especies y su capacidad competitiva le permite a las especies menos competitivas colonizar los sitios dejados libres por las especies más competitivas, pero también más limitadas en su dispersión (Tilman, 1994; Hurtt \& Pacala, 1995). De esta manera, la ganadora entre dos especies que compiten localmente no es necesariamente la mejor competidora de la comunidad sino la mejor competidora que logró llegar a un micrositio en particular. Esto es lo que varios autores han llamado winning by forfeit (ganar por chance), dado que la llegada de una 
semilla a un micrositio tiene un fuerte componente de azar. Es por esto que la dispersión de semillas es frecuentemente asociada con los procesos aleatorios (Hurtt \& Pacala, 1995; Hubbell, 2001).

Son muchos los estudios que han medido la limitación en el reclutamiento por ausencia de llegada de semillas. Una manera de hacer esto consiste en adicionar semillas de forma experimental. Si las especies están limitadas en su dispersión, entre más semillas lleguen a un micrositio, mayor será su reclutamiento (Figuras 1a y 2a). Turnbull et al. (2000) realizaron una revisión bibliográfica de los estudios publicados sobre este tema y concluyeron que, para aproximadamente la mitad de las poblaciones estudiadas, la probabilidad

(a)

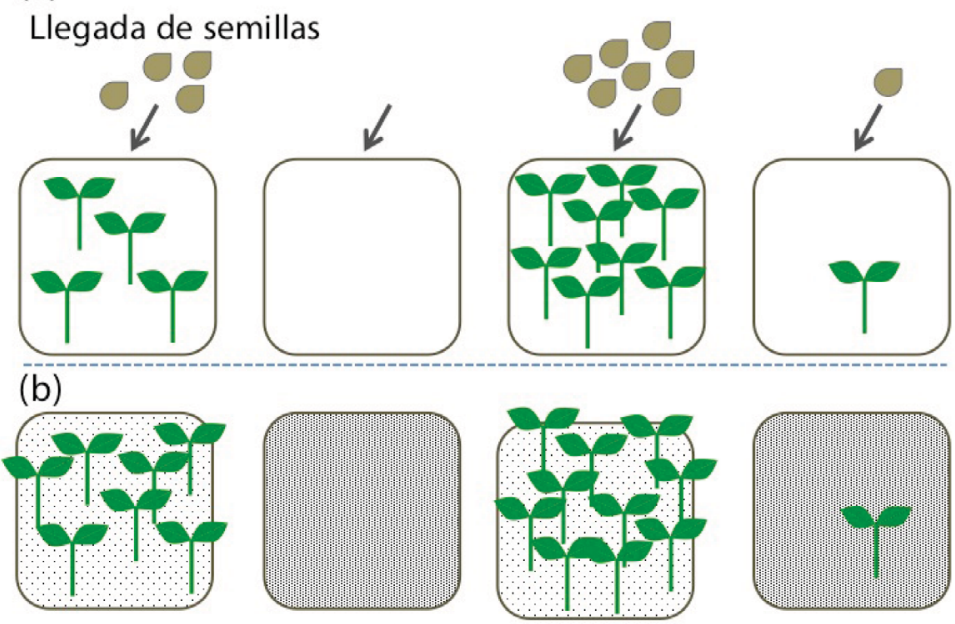

Heterogeneidad ambiental

Figura 1. Ilustración de los procesos que generan una limitación en el reclutamiento de plántulas.

(a) La llegada de las semillas a un micrositio en particular es el principal factor que determina el reclutamiento de plántulas.(b) Los distintos patrones representan distintas condiciones abióticas.Las preferencias de hábitat de una especie dada limitan el reclutamiento de plántulas a los micrositios en donde las condiciones ambientales son favorables para la especie

(a)

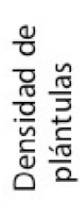

(a)

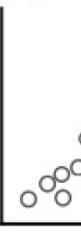

(b)

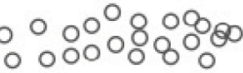

(c)

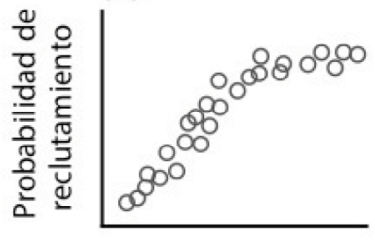

Calidad del hábitat
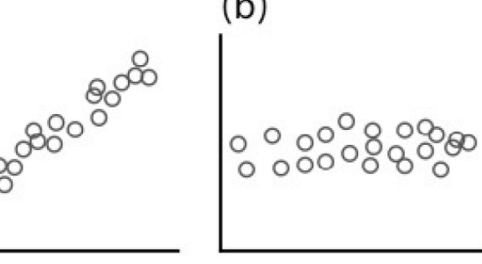

Densidad de semillas

(d)

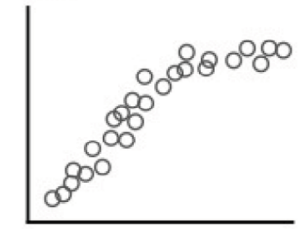

Distancia al conspecífico adulto más cercano (e)

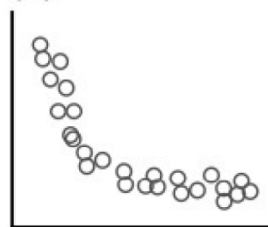

Densidad de conspecíficos

Figura 2. Relación entre densidad de semillas y densidad de plántulas $(a, b)$ y probabilidad de establecimiento según el procesos ecológico que predomina (c, d, e).

Si el reclutamiento está limitado principalmente por la llegada de semillas, se espera una relación positiva entre densidad de semillas y de plántulas (a). Si los factores ambientales representan el principal filtro ecológico durante el reclutamiento, no se espera ninguna relación en particular entre densidad de semillas y de plántulas (b). En cambio, la probabilidad de reclutamiento debería aumentar cuando la calidad de hábitat aumenta (c). En caso de distancia-dependencia negativa, la probabilidad de reclutamiento aumenta a medida que la distancia al conspecífico adulto más cercano aumenta (d). En caso de la densidad-dependencia negativa, la probabilidad de reclutamiento disminuye a medida que la densidad de conspecíficos aumenta (e). 
de reclutamiento aumenta cuando se elimina el filtro de llegada de semillas (i.e. se agregan semillas, Figura 1a). Sin embargo, la mayoría de estos estudios fueron realizados en zonas templadas, en donde la diversidad de plantas no es muy alta. En bosques tropicales es difícil realizar este tipo de aproximaciones experimentales, teniendo en cuenta un número de especies que sea representativo de la diversidad que caracteriza dichos ecosistemas. Entre los pocos estudios que han hecho esto, Paine \& Harms (2009) sembraron experimentalmente semillas de ocho especies de árboles en distintos tratamientos de densidad y diversidad en el PNN de Cocha Cashu (Perú). Sus resultados mostraron que la adición de semillas afecta considerablemente la composición de especies de plántulas, lo que confirma que la llegada de semillas a un micrositio dado es un filtro ecológico muy importante durante el reclutamiento de plántulas.

Otra manera de medir la limitación en la dispersión es estimando la proporción de sitios a los que llegan regularmente semillas de una especie en particular (Muller-Landau et al., 2002). El establecimiento de trampas de semillas en parcelas permanentes permite describir de manera más general los patrones de lluvia de semillas para una comunidad de árboles. El estudio más completo en este campo se ha llevado a cabo en Barro Colorado Island (BCI, Panamá), en donde una red de 200 trampas de semillas fue establecida en una parcela de 50 ha en 1985 (Wright \& Calderón, 1995). Desde entonces, todas las semillas y frutos que caen en las trampas son colectadas cada quince días (Wright et al., 2005). Este estudio ha permitido comprender la variabilidad temporal en los patrones de lluvia de semillas (Wright et al., 2005) y ha demostrado que la mayoría de las especies de árboles están severamente limitadas en su dispersión (Hubbell et al., 1999). De las 260 especies de árboles registradas en la parcela, más del $80 \%$ fracasaron en dispersar sus semillas en al menos una de las 200 trampas en un periodo de diez años (Hubbell et al., 1999). Hoy en día, esta metodología ha sido ampliamente replicada en varios bosques de países tropicales y sub-tropicales, como es el caso de Ecuador (Metz et al., 2008), Guyana Francesa (Norden et al. 2007a, Norden et al., 2009a), Puerto
Rico (Muscarella et al. 2013), Malasia (Metz et al., 2008), Hawaii (Inman-Narahari, 2013) y China (Du et al., 2012). De manera general, todos estos estudios convergen en encontrar que la dispersión de las especies de árboles es altamente limitada.

\section{DIFERENCIACIÓN DE NICHOS DURANTE LA FASE DE REGENERACIÓN}

Una vez el filtro de la dispersión es superado, la llegada de una semilla a un micrositio no garantiza el reclutamiento exitoso de la plántula (Figuras $\underline{1 b}$ y $\underline{2 b}$ ). Otros filtros ecológicos, esta vez de índole ambiental, pueden impedir que la semillas germinen y/o que persistan en el sotobosque. Lo anterior es más marcado si las especies muestran preferencia hacia algún hábitat en particular. Grubb (1977) propuso que las condiciones ambientales necesarias para el establecimiento de las plantas en el sotobosque varían según las especies, lo que implica que la partición de los recursos, o diferenciación de nichos entre las especies, comienza muy temprano en el ciclo de vida de las plantas (Figuras. 1b y 2c $)$.

Inicialmente, los estudios sobre la partición de recursos entre las especies durante la fase de regeneración se focalizaron en el rol de los claros en el bosque (Brokaw, 1985; Denslow, 1987; Nicotra et al., 1999; Montgomery \& Chazdon, 2002). Sin embargo, los claros afectan tan solo el $1 \%$ de la cobertura boscosa cada año (Hartshorn, 1978; Riéra, 1983) y la frecuencia de distribución de la luz está fuertemente sesgada hacia sitios que reciben menos del $2 \%$ de las radiaciones luminosas totales (Montgomery \& Chazdon, 2002). En consecuencia, el paradigma claros versus sotobosque ignora el papel de luz en el establecimiento de las plántulas en la mayor parte del gradiente luminoso, y se limita a explicar únicamente la coexistencia entre especies pioneras y especies tolerantes a la sombra. En realidad, la mayoría de las especies de árboles tropicales son tolerantes a la sombra, y emplean diversas estrategias para crecer y sobrevivir en bajos niveles de luz (Kitajima, 1994, 1996; Montgomery \& Chazdon, 2002). En los bosques tropicales secos la discontinuidad del dosel resulta 
en una alta disponibilidad de luz en el sotobosque, por lo cual las especies de árboles tropicales no responden de manera muy variable a los distintos niveles de luz (Vargas-Rodríguez et al., 2005, Markesteijn et al., 2007). De manera general, aunque la luz sí es probablemente un factor limitante que ejerce una presión de selección en las especies de árboles tropicales, no parece ser la mayor fuente de diferenciación de nichos durante el proceso de regeneración.

Los factores edáficos, en cambio, pueden tener un efecto más importante que la luz sobre la distribución espacial de las especies en los bosques tropicales (Sollins, 1998; Figura 1b). Entre las características más importantes del suelo se encuentran la disponibilidad de fósforo y nitrógeno asimilables, el $\mathrm{pH}$ y la disponibilidad de agua, que a su vez depende de la porosidad del suelo y de la profundidad de la capa freática. Varios estudios experimentales en bosques húmedos han mostrado asociaciones de las especies de plántulas a distintos tipos de suelo en condiciones muy contrastantes (Hall et al., 2003; Fine et al., 2004; Palmiotto et al., 2004). Otros estudios en bosques secos muestran que los nutrientes del suelo, en particular el fósforo, tienen un efecto positivo en la supervivencia y crecimiento de las plántulas (Vargas-Rodríguez et al., 2005; Ceccon et al., 2007; Campo \& VásquezYañez, 2004). Sin embargo, estos patrones no son tan conspicuos a lo largo de gradientes edáficos menos marcados y las condiciones del suelo no tienen un poder explicativo muy alto en la distribución y abundancia relativa de varias especies de plántulas (Webb \& Peart, 2000; Baraloto \& Goldberg, 2004; Norden, et al. 2009b).

Uno de los factores edáficos más limitantes para las plantas es el agua. La relación entre los patrones de distribución y abundancia relativa de las especies con la precipitación y la disponibilidad de agua en el suelo ha sido ampliamente documentada (Gentry, 1988; Swaine 1996; Bongers et al., 1999). Engelbrecht et al. (2007) mostraron que, en Panamá, la diferenciación de nichos con respecto a la disponibilidad de agua en el suelo determina la abundancia relativa de las especies de árboles a lo largo del canal de Panamá, en donde hay un gradiente de precipitación muy marcado. Las plántulas de las especies más sensibles a la sequía fueron mucho más abundantes en zonas húmedas, mientras que aquellas más resistentes a largos períodos de sequía fueron más abundantes en los bosques estacionales. Estos patrones se repitieron a escalas locales, en donde la topografía (la cual determina la variación local en la disponibilidad de agua en el suelo) fue determinante en la distribución espacial de las plántulas.

Si la limitación en el reclutamiento es el resultado de preferencias de hábitat por parte de las especies y no de una limitación en la dispersión, entonces los patrones de distribución de plántulas deberían estar altamente correlacionados con los factores ambientales y no con la llegada de semillas (Figuras $\underline{1 b}, \underline{2 b y ~ 2 c)}$. Sin embargo, los patrones de asociación de hábitat en las especies de árboles tropicales son generalmente más notorios en adultos que en plántulas y juveniles (Harms, et al. 2001; Condit et al., 2013). Esto se debe probablemente a que el filtro ambiental que genera la asociación de una especie a un hábitat en particular actúa lentamente a lo largo de la ontogenia (Webb \& Peart, 2000). De hecho, varios estudios han mostrado que las preferencias de hábitat de las especies pueden cambiar a lo largo del tiempo (Webb \& Peart 2000; Comita et al. 2007). Esto sugiere que las asociaciones de hábitat observadas en adultos no son necesariamente el resultado de una diferenciación de nichos durante la fase de regeneración.

\section{VARIABILIDAD TEMPORAL EN EL RECLUTAMIENTO}

Chesson \& Warner (1981) mostraron que las fluctuaciones en el reclutamiento también pueden promover la coexistencia de las especies. Teóricamente, si las especies abundantes y las especies raras no son reclutadas de manera sincrónica, la probabilidad de éxito de las especies raras aumenta dado que así evitan la competencia directa con las especies abundantes. Este efecto de la cohorte integra una perspectiva temporal a teorías basadas en componentes puramente espaciales (Chesson \& Warner, 1981; Warner \& Chesson, 1985; Chesson, 2000). Fluctuaciones 
temporales en distintos procesos ecológicos como la producción de frutos (Wright et al., 1999; Wright \& Calderón, 2005; Norden et al., 2007b) o la abundancia de polinizadores (Bawa, 1990) y dispersores de semillas (Stevenson, 2004), pueden crear una partición temporal de los nichos ecológicos, reduciendo la competencia entre las especies y por ende facilitando el mantenimiento de la biodiversidad. Tales fluctuaciones pueden ser deterministas si son el resultado, por ejemplo, de una estacionalidad en el clima, o estocásticas, si muestran un carácter irregular (Chesson, 2000). En los bosques tropicales las especies muestran una gran variedad de patrones fenológicos en la producción de semillas (Sakai, 2001) y responden de manera muy diferente a las variables o eventos climáticos tales como el Niño (Wright \& Calderón, 1995, Curran et al., 1999). Por otro lado, la producción de semillas puede variar en el tiempo y el espacio entre los individuos de una misma población (Itoh et al., 2003). Todas estas fuentes de variación provocan una fluctuación temporal muy importante en el reclutamiento de plántulas (Forget, 1997; Connell \& Green, 2000), y hacen que la estocasticidad sea parte del proceso de regeneración.

Kelly \& Bowler (2002) demostraron en un bosque seco de México, que la coexistencia de especies de plántulas congéneres se da por este tipo de proceso. Dichos autores concluyen que el efecto de la cohorte tiene un impacto muy importante en la coexistencia de especies. Desafortunadamente, esta hipótesis es prácticamente imposible de probar en árboles, dado que necesitaría datos empíricos a largo plazo sobre la dinámica de las poblaciones que coexisten durante varias generaciones.

\section{DENSIDAD-DEPENDENCIA NEGATIVA}

Otro mecanismo posible para explicar la coexistencia de especies en los bosques tropicales, y cuyo efecto ocurre durante la fase de regeneración, es la densidad o distanciadependencia negativa (DDN). La hipótesis que invoca estos procesos fue propuesta de manera independiente por Janzen (1970) y Connell (1971) y propone que la supervivencia de las plántulas se reduce a proximidad de adultos de la misma especie debido al ataque de insectos herbívoros y hongos patógenos altamente especialistas. En otras palabras, los enemigos naturales de las plantas limitan el reclutamiento de individuos conspecíficos al tiempo que favorecen el de individuos de otras especies, según la distancia de adultos de la misma especie o la densidad de plántulas conspecíficas. Lo anterior se da a partir del ataque a semillas (Paine \& Beck, 2007) o a plántulas (Augspurger,1984; Fig. 2d,e). De esta manera, la DNN en un mecanismo estabilizante que promueve la coexistencia de las especies (Chesson 2000).

La DNN es reconocida como uno de los principales mecanismos que contribuyen al mantenimiento de la diversidad en los bosques tropicales. Desde la publicación de la teoría de Janzen-Connell, hace 40 años, casi 2000 estudios han probado sus predicciones en múltiples bosques tropicales y en cientos de especies (Comita et al., 2014). Aunque la literatura concuerda en que la DDN prevalece en los bosques tropicales (Clark \& Clark, 1984; Terborgh, 2012), la proporción de especies que sufren de DDN varía según los estudios y no es claro hasta qué punto este es un mecanismo generalizado en las comunidades de árboles tropicales. Un meta-análisis reciente, que incluyó datos de más de 60 publicaciones, encontró soporte para las predicciones de Janzen (1970) y Connell (1971), y mostró que, de manera general, la probabilidad de supervivencia disminuye significativamente cuando los individuos se encuentran cerca a conspecíficos comparado a cuando están cerca de heteroespecíficos (Comita et al., 2014).

Aunque la evidencia a favor de la DDN es muy fuerte, la gran mayoría de estos estudios no demuestra directamente que los responsables de este proceso son los enemigos naturales de las plantas. Tan solo recientemente el efecto de herbívoros y patógenos ha sido evaluado de manera directa. Mangan et al. (2010) mostraron experimentalmente en Barro Colorado Island (BCI, Panamá) que existe una respuesta diferencial de las plántulas a la biota del suelo, según la proveniencia de esta. El crecimiento de plántulas de cuatro especies de árboles se vio reducido cuando el suelo en el que 
crecían fue inoculado por biota del suelo colectada debajo de adultos conspecíficos, en comparación con inoculaciones colectadas debajo de árboles de otras especies. Los resultados muestran claramente la existencia de una fuerte interacción negativa y especializada entre las plantas y la biota del suelo. Bagchi et al. (2013) utilizaron insecticidas $\mathrm{y}$ fungicidas en un bosque tropical de Belice para evaluar directamente el efecto de la ausencia de enemigos naturales en las plántulas y demostraron que bajo este tratamiento, la diversidad de plántulas disminuye y la composición de las especies cambia drásticamente. Juntos, estos estudios demuestran que las interacciones bióticas son una fuerza muy importante en la estructuración de las comunidades de plantas y que actúan principalmente durante la fase de regeneración.

Desde un punto de vista teórico, la DDN contribuye a la coexistencia únicamente si es un mecanismo que tiene un efecto "compensatorio", es decir, si son las especies más abundantes aquellas que sufren una DDN más fuerte. Un estudio en el PNN Yasuní (Ecuador) mostró que la tasa de supervivencia de las plántulas de varias especies de Myristicaceae estaba negativamente correlacionada con la abundancia relativa de los árboles (Queenborough et al., 2007). Esto sugiere que las especies más comunes están reguladas desde las fases tempranas en el ciclo de vida de las plántulas. En BCI (Panamá), varios estudios coinciden en que son justamente las especies menos abundantes como adultos aquellas que sufren la DDN más fuerte (Comita et al., 2010; Mangan et al., 2010). Esto sugiere que las especies raras lo son porque ya han pasado por una DDN durante las fases más tempranas del ciclo de vida.

Pese a la gran atención que ha recibido, muchas preguntas quedan aún por resolver sobre la importancia de la DDN para la coexistencia de las especies. Por ejemplo, si este mecanismo es uno de los mayores promotores de la coexistencia de especies en los bosques tropicales, se espera que sea más intenso y ocurra en una mayor proporción de especies en zonas tropicales. Sin embargo, estudios sobre regeneración en zonas templadas también evidencian una fuerte DDN en muchas especies de árboles (Hille Ris Lambers et al., 2002). Comita et al. (2014) tampoco encontraron un efecto de la latitud en la intensidad de este mecanismo (Comita et al., 2014). Más estudios experimentales que demuestren el efecto de los enemigos naturales en la regulación de la abundancia relativa de las especies de árboles deben ser reproducidos en otros bosques en regiones tropicales y templadas.

\section{CONCLUSIONES}

Los patrones de distribución y abundancia relativa de las especies de árboles en los bosques tropicales son probablemente el resultado de distintos filtros ecológicos que ocurrieron en el pasado, durante la fase de regeneración. La limitación en la dispersión, los filtros ambientales, la variabilidad temporal en factores bióticos y abióticos y la densidad-dependencia negativa son todos factores que obstaculizan el reclutamiento de las plántulas en el sotobosque, lo que hace de la transición semilla-plántula la más difícil de todo el ciclo de vida de las plantas (Muller-Landau et al., 2002). Algunos de estos procesos le dan un papel preponderante al determinismo ecológico, pues se basan en las interacciones bióticas y abióticas de las plantas con su entorno (diferenciación de nichos y DDN). Otros, en cambio, le dan más importancia a la deriva ecológica, pues incluyen el azar en la dispersión de los propágulos (limitación en la dispersión y variabilidad temporal). Esta combinación de determinismo y estocasticidad durante el proceso de regeneración es determinante para la coexistencia de las especies (Hurtt \& Pacala, 1995). Si las especies no estuvieran limitadas en su reclutamiento, únicamente ocuparían el nicho en donde son las mejores competidoras (nicho realizado). Sin embargo, la heterogeneidad espaciotemporal que caracteriza los bosques tropicales no genera los nichos ecológicos suficientes para la coexistencia de las miles de especies que encontramos en estos ecosistemas. Al estar tan limitadas en su reclutamiento, las especies tienen la oportunidad de ocupar un nicho más amplio, dado que son capaces de colonizar nuevos sitios en la ausencia de competidores (Hurtt \& Pacala, 1995). De esta manera, incluso cuando las diferencias competitivas entre las especies son importantes, la deriva ecológica que resulta de la variabilidad 
temporal de diversos factores bióticos y abióticos y del azar en la dispersión de las semillas contribuye al mantenimiento de la diversidad.

Sin embargo, todavía queda mucho por aprender sobre la importancia de la regeneración en los bosques tropicales. Son necesarios más estudios que incluyan un número de especies que sea representativo de la biodiversidad que caracteriza estos biomas y que tengan en cuenta distintas escalas espaciales, a fin de comprender la magnitud de la limitación en el reclutamiento a nivel de la comunidad de árboles. Igualmente, datos a largo plazo que permitan cuantificar la variabilidad temporal del éxito en el reclutamiento son esenciales para tener una aproximación más precisa de la dinámica de la comunidad de plántulas en el sotobosque, y de cómo esta afecta los patrones de distribución y abundancia en los árboles.

En los bosques que han sufrido perturbaciones, la regeneración permite recuperar la estructura original, y gran parte de la flora y fauna perdida (Aide \& Grau, 2004; Dent \& Wright, 2009; Norden et al., 2009b). Dado que este proceso depende principalmente de la llegada de propágulos colonizadores y de las condiciones abióticas en donde se regenera el bosque (Chazdon, 2003), todos los procesos ecológicos mencionados juegan un papel determinante en la recuperación de los bosques. Si la perturbación no ha sido muy fuerte y el bosque secundario que regenera hace parte integrante de un paisaje que cuenta con parches de bosque maduro, se espera que la limitación en la dispersión y los filtros ambientales no sean muy severos. En dichos casos, muchos atributos de la estructura (por ejemplo: densidad de palos, área basal y riqueza específica) de los bosques secundarios se recuperan en cuestión de algunas décadas (Chazdon, 2003). En cambio, si los bosques sucesionales se encuentran aislados de otros remanentes de bosque, es más difícil que recuperen la estructura y diversidad originales (Chazdon, 2003; Norden et al., 2011). Entender cuáles son las principales fuerzas que limitan el reclutamiento de plántulas en los bosques con algún nivel de degradación es fundamental para poder tener un poder predictivo sobre la trayectoria sucesional que han de seguir estos ecosistemas. Esto permitiría definir las estrategias de conservación adecuadas para la conservación de los distintos tipos de bosque que se encuentran en las regiones tropicales. Por ejemplo, si la limitación en la dispersión es el principal filtro ecológico en la sucesión de un bosque determinado, la introducción de propágulos de especies nativas puede ser una manera apropiada para manipular o acelerar la dinámica sucesional (Young et al., 2005). En otros casos, el filtro ambiental puede ser la mayor limitante para el reclutamiento exitoso de las plántulas (por ejemplo la minería), en cuyo caso es necesario realizar un mejoramiento del suelo previo a la introducción de propágulos. Ahondar en estos temas es fundamental para una valoración integral de los ecosistemas que conduzca al planteaminento de planes de manejo eficaces para la conservación y el uso sostenible de los ecositemas tropicales.

\section{AGRADECIMIENTOS}

Esta revisión bibliográfica es uno de los productos de mi investigación doctoral. Agradezco a Jérôme Chave por sus contribuciones a lo largo de este proceso. Camila Pizano y Tim Paine brindaron importantes comentarios que aportaron al texto.

\section{REFERENCIAS BIBLIOGRÁFICAS}

Aide, T.M., \& Grau, H.R. (2004). Globalization, migration and Latin American ecosystems. Science, 305, 1915-1916.

Augspurger, C.K. (1984). Seedling survival of tropical tree species: Interactions of dispersal distance, light-gaps, and pathogens. Ecology, 65, 1705-1712.

Baraloto, C., \& Goldberg, D.E. (2004). Microhabitat associations and seedling bank dynamics in a neotropical forest. Oecologia, 141, 701-712.

Bagchi, R., Gallery, R.E., Gripenberg, S., Gurr, S.J., Narayan, L., Addis, C.E., Freckleton, R.P., \& Lewis, O.T. (2014). 
Pathogens and insect herbivores drive rainforests plant diversity and composition. Nature, 506, 85-88.

Bawa, K.S. (1990). Plant-pollinator interactions in tropical rain forests. Annual Review of Ecology and Systematics, 21, 399-422.

Bongers, F., Poorter, L., Rompaey, R., \& Parren, M.P.E. (1999). Distribution of twelve moist forest canopy tree species in Liberia and Cote d'Ivoire: response curves to a climatic gradient. Journal of Vegetation Science, 10, 371-382.

Brokaw, N.V.L. (1985) Gap-phase regeneration in a tropical forest. Ecology, 66, 682-687.

Chazdon, R.L. (2003). Tropical forest recovery: legacies of human impact and natural disturbancies. Perspectives in Plant Ecology, Evolution and Systematics, 6, 5171.

Ceccon, E., Sanchez, S., \& Campo, J. (2004) Tree seedling dynamics in two abandoned tropical dry forests of differing successional status in Yucatán, Mexico: a field experiment with $\mathrm{N}$ and $\mathrm{P}$ fertilization, Plant Ecology, 170, 277-285.

Chesson, P. (2000) General theory of competitive coexistence in spatially-varying environments. Theoretical Population Biology, 58, 211-237.

Chesson, P.L., \& Warner, R.R. (1981) Environmental variability promotes coexistence in lottery competitive-systems. American Naturalist, 117, 923-943.

Campo, J., \& Vásquez-Yanez, C. (2004). Effects of nutrient limitation on aboveground carbon dynamics during tropical dry forest regeneration in Yucatán, Mexico, Ecosystems, 7, 311-319.
Clark, D.A., \& Clark, D.B. (1984). Spacing dynamics of a tropical rain forest tree: evaluation of the Janzen-Connell model. American Naturalist, 124, 769-788.

Comita, L.S., Condit, R., \& Hubbell, S.P. (2007). Developmental changes in habitat associations of tropical trees. Journal of Ecology, 95, 482, 492

Comita, L.S., Muller-Landau, H.C., Aguilar, S., \& Hubbell, S.P. (2010) Asymmetric density-dependence shapes species abundances in a tropical tree community. Science, 329: 330-332.

Comita, L.S., Queenborough, S.A., Murphy, S.J., Eck, J.L., Kaiyang, X., Krishnadas, M., Beckman, N., \& Zhu, Y. (2014). Testing predictions of the JanzenConnell hypothesis: a meta-analysis of experimental evidence for distance and density-dependent seed and seedling survival. Journal of Tropical Ecology, 102, 845-856.

Condit, R., Engelbrecht, B. M. J., Pino, D., Pérez, R., \& Turner, B.L. (2013). Species distributions in response to individual soil nutrients and seasonal drought across a community of tropical trees. Proceedings of the National Academy of Sciences, 110, 5064-5068.

Connell, J.H. (1971) On the role of natural enemies in preventing competitive exclusion in some marine animals and in rain forest trees. En P.J. den Boer, G.R. Gradwell (eds.) Dynamics of populations. Pudoc, Wageningen, pp. 298-312.

Connell, J.H. \& Green, P.T. (2000) Seedling dynamics over thirty-two years in a tropical rain forest tree. Ecology, 81, 568-584.

Curran, L.M., Caniago, I., Paoli, G.D., Astianti, D., Kusneti, M., Leighton, M., Nirarita, 
C.E. \& Haeruman, H. (1999) Impact of El Nino and logging on canopy tree recruitment in Borneo. Science, 286, 21842188.

Denslow, J.S. (1987). Tropical rainforest gaps and tree species diversity. Annual Review of Ecology and Systematics, 18, 431-451.

Dent, D.H., \& Wright, S.J. (2009). The future of tropical species in secondary forests: A quantitative review. Biological conservation, 142, 2833-2843.

Du, Y., Mi, X., Liu, X., \& Ma, K. (2012). The effects of ice storm on seed rain and seed limitation in an evergreen broad-leaved forest in east China. Acta Ecologica, 39, 87-93.

Engelbrecht, B.M.J., Comita, L.S., Condit, R., Kursar, T.A., Tyree, M.T., Turner, B.L., \& Hubbell, S.P. (2007). Drought sensivity shapes species distribution patterns in tropical forests. Nature, 447, 80-83.

Fine, P.V.A., Mesones, I. \& Coley, P.D. (2004) Herbivores promote habitat specialization by trees in Amazonian forests. Science, 305, 663-665.

Forget, P.-M. (1997). Ten-year seedling dynamics in Vouacapoua americana in French Guiana: A hypothesis. Biotropica, 29, 124126.

Gentry, A.H. (1988). Changes in plant community diversity and flo- ristic composition on environmental and geographical gradients. Annual Misouri Botanical Garden, 75, $1-34$

Grubb, P.J. (1977). Maintenance of SpeciesRichness in Plant Communities Importance of Regeneration Niche. Biological Reviews of the Cambridge Philosophical Society, 52, 107-145.
Hall, J.S., Ashton, P.M.S. \& Berlyn, G.P. (2003) Seedling performance of four sympatric Entandrophragma species (Meliaceae) under simulated fertility and moisture regimes of a Central African rain forest. Journal of Tropical Ecology, 19, 55-66.

Harms, K.E., Condit, R., Hubbell, S.P., \& Foster, R.B. (2001). Habitat associations of trees and shrubs in a 50-ha neotropical forest plot. Journal of Ecology, 89, 947959.

Harms, K.E., Wright, S.J., Calderón, O., Hernández, A. \& Herre, E.A. (2000). Pervasive density-dependent recruitment enhances seedling diversity in a tropical forest. Nature, 404, 493-495.

Hartshorn, G.S. (1978). Tree falls and forest dynamics. En P.B. Tomlinson \& M.H. Zimmerman (eds.) Tropical Trees and Living Systems pp 617-638, Cambridge University Press.

Hille Ris Lambers, J., Clark, J.S. \& Beckage, B. (2002). Density-dependent mortality and the latitudinal gradient in species diversity. Nature, 417, 732-735.

Howe, H.F., Schupp, E.W., \& Westley, L.C. (1985). Early consequences of seed dispersal for a neotropical tree (Virola surinamensis). Ecology, 66, 781-791.

Howe, H.F. \& Smallwood, J. (1982). Ecology of Seed Dispersal. Annual Review of Ecology and Systematics, 13, 201-28.

Howe, H.F. \& Vande Kerckhove, G.A. (1981). Removal of wild nutmeg Virola surinamensis crops by birds. Ecology, 62, 1093-1106.

Hubbell, S.P. (1980). Seed Predation and the Coexistence of Tree Species in Tropical Forests. Oikos, 35, 214-229. 
Hubbell, S.P., He, F., Condit, R., Borda-deAgua, L., Kellner, J., \& ter Steege, H. (2008). How many species are there in the Amazon and how many of them will go extinct? Proceedings of the National Academy of Sciences, 105, 11498-11504.

Hubbell, S.P. (2001) The Unified Neutral Theory of Biodiversity and Biogeography. Princeton University Press, Princeton.

Hubbell, S.P., Foster, R.B., O'Brien, S.T., Harms, K.E., Condit, R., Wechsler, B., Wright, S.J. \& de Lao, S.L. (1999). Lightgap disturbances, recruitment limitation, and tree diversity in a neotropical forest. Science, 283, 554-557.

Hurtt, G.C. \& Pacala, S.W. (1995). The consequences of recruitment limitation - Reconciling chance, history and competitive differences between plants. Journal of Theoretical Biology, 176, 1-12.

Inman-Narahari, F., Ostertag, R., Cordell, S., Giardina, C., Nelson-Kaula, K., \& Sack, L. (2013). Seedling recruiment factors in low-diversity Hawaiian wet forest towards global comparisons among tropical forests. Ecosphere, 4, art24.

Itoh, A., Yamakura, T., Ohkubo, T., Kanzaki, M., Palmiotto, P., Tan, S. \& Lee, H.D. (2003). Spatially aggregated fruiting in an emergent Bornean tree. Journal of Tropical Ecology, 19, 531-538.

Janzen, D.H. (1969) Seed-eaters versus seed size, number, toxicity and dispersal. Evolution, $23,1-27$.

Janzen, D.H. (1970). Herbivores and the number of tree species in tropical forests. American Naturalist, 104, 501-528.

Kelly, C.K., \& Bowler, M.G. (2002). Coexistence and relative abundance in forests trees. Nature, 417, 437-440.
Kitajima, K. (1994). Relative importance of photosynthetic traits and allocation patterns as correlates of seedling shade tolerance of 13 tropical trees. Oecologia, 98, 419-428.

Kitajima, K. (1996). Ecophysiology of tropical tree seedlings. En S.S. Mulkey (ed.) Tropical Forest Plant Ecophysiology, pp. 559-596, Chapman and Hall, New York

Lambin, E.F., \& Geist, H. (2006). Land use and land cover change: Local processes and global impacts. The IGBP Series. Springer-Verlag, Berlin, Germany.

Laurance, W.F. (2007). Have we overstated the tropical biodiversity crisis? Trends in Ecology and Evolution, 22, 65-70.

Lewis, S.L., Malhi, Y., \& Phillips, O.L. (2004). Fingerprinting the impacts of gloval change on tropical forests. Philosophical Transactions of the Royal Society of London Series B, 359, 437-462.

Mangan, S.A., Schnitzer, S.A., Herre, E.A., Mack, K.M.L., Valencia, M.C., Sánchez, E.I., \& Bever, J.D. (2010). Negative plant-soil feedback predicts tree-species relative abundance in a tropical forest. Nature, 466, 752-756.

Markesteijn, L. Poorter L, Bongers F. (2007). Light-dependent leaf-trait variation in 43 tropical dry forests. American Journal of Botany, 94, 515-525.

Masaki, T., Tanaka, H., Tanouchi, H., Sakai, T. \& Nakashizuka, T. (1999). Structure, dynamics and disturbance regime of temperate broad-leaved forests in Japan. Journal of Vegetation Science, 10, 805814.

Melillo, J.M., Mcguire, A.D., Kicklighter, D.W., Moore Iii, B., Vorosmarty, C.J., \& Schloss AL. (1993). Global climate change 
and terrestrial net primary production. Nature, 363, 234-240.

Metz, M.R., Comita, L.S., Chen, Y.Y., Norden, N., Condit, R., Hubbell, S.P, FangSung, I., Noor, Md., \& Wright, J.S. (2008) Temporal and spatial variability in seedling dynamics: a cross site comparison in four lowland tropical forests Journal of Tropical Ecology, 24, 9-18

Montgomery, R.A. \& Chazdon, R.L. (2002). Light gradient partitioning by tropical tree seedlings in the absence of canopy gaps. Oecologia, 131, 165-174.

Muller-Landau, H.C., Wright, S.J., Calderón, O., Hubbell, S.P., \& Foster, R.B. (2002). Assessing recruitment limitation: concepts, methods, and case-studies from a tropical forest. En D.J. Levey, W.R. Silva, \& M. Galetti (eds.) Seed Dispersal and Frugivory: Ecology, Evoluation and Conservation pp. 35-53, CAB International, Wallingford, Oxfordshire.

Muscarella, R., Uriarte, M., Forero-Montaña J., Comita, L.S., Swenson, N., Thompson, J., Nytch, C.J., Jonckheere, I., \& Zimmerman, J.K. (2013). Life-history trade-offs during the seed-to-seedling transition in a subtropical wet forest community. Journal of Ecology, 101, 171.182 .

Nathan, R., \& Muller-Landau, H.C. (2000). Spatial patterns of seed dispersal, their determinants and consequences for recruitment. Trends in Ecology \& Evolution, 15, 278-285.

Nicotra, A.B., Chazdon, R.L., \& Iriarte, S.V.B. (1999). Spatial heterogeneity of light and woody seedling regeneration in tropical wet forests. Ecology, 80, 1908-1926.

Norden, N., Chave, J., Caubere, A., Chatelet, P., Ferroni, N., Forget, P.-M., \& Thebaud,
C. (2007a). Is temporal variation of seedling communities determined by environment or by seed arrival? A test in a neotropical forest. Journal of Ecology, 95, 507-516

Norden, N., Chave, J., Belbenoit, P., Caubere, A., Chatelet, P., Forget, P.-M., \& Thebaud, C. (2007b). Mast Fruiting Is a Frequent Strategy in Woody Species of Eastern South America. Plos One 2(10): e1079.

Norden, N., Chave, J., Belbenoit, P., Caubere, A., Chatelet, P., Forget, P.-M., Riera, B., Viers, J., \& Thebaud, C. (2009a). Interspecific variation in seedling responses to seed limitation and habitat conditions for 14 Neotropical woody species. Journal of Ecology 97, 186-197.

Norden, N., Chazdon, R.L., Chao, A., Jiang, Y.H., \& Vílchez-Alvarado, B. (2009b). Resilience of tropical rain forests: tree community reassembly in secondary forests. Ecology Letters, 12, 385-394.

Norden, N., Mesquita, R.C.G., Bentos, T.V., Chazdon, R.L., \& Williamson, G.B. (2011). Contrasting community compensatory trends in alternative successional pathways in Central Amazonia. Oikos, 120, 143-151.

Paine, C.E.T., \& Harms, K.E. (2009) Quantifying the effects of seed arrival and environmental conditions of tropical seedling community structure. Oecologia, 160, 139-150.

Paine, C.E.T., \& Beck, H. (2007). Seed predation by Neotropical rainforest mammals increases divserity in seedling recruitment. Ecology, 88, 3076-3087.

Palmiotto, P.A., Davies, S.J., Vogt, K.A., Ashton, M.S., Vogt, D.J. \& Ashton, P.S. (2004) Soil-related habitat specialization 
in dipterocarp rain forest tree species in Borneo. Journal of Ecology, 92, 609-623.

Pitman, N.C.A., Terborgh, J.W., Silman, M.R., Nunez, P., Neill, D.A., Cerón, C.E., Palacios, W.A. \& Aulestia, M. (2001) Dominance and distribution of tree species in upper Amazonian terra firme forests. Ecology, 82, 2101-2117.

Pitman, N.C.A., Terborgh, J.W., Silman M.R., Nunez, P., Neill, D.A., Cerón, C.E., Palacios, W.A. \& Aulestia, M. (2002). A comparison of tree species diversity in two upper Amazonian forests. Ecology, 83, 3210-3224.

Prance, G.T. (1977). Floristic inventory of tropics: where do we stand? Annals of the Missouri Botanical Garden, 64, 659-684.

Prance, G.T., Beentje, H., Dransfield, J., \& Johns, R. (2000). The tropical flora remains undercollected. Annals of The Missouri Botanical Garden, 87, 67-71.

Queenborough, S.A., Burslem, D.F.R.P., Garwood, N., \& Valencia, R. (2007). Neighborhood and community interactions determine the spatial pattern of tropical tree seedling survival. Ecology, 88, 2248-2258.

Ratiarison, S. (2003). Frugivorie dans la canopée de la forêt guyanaise : conséquences pour la pluie de graines. Thèse de Doctorat, Université Paris 6, Paris.

Riéra, B. (1983) Chablis et cicatrisation en forêt guyanaise (Piste Saint-Elie), Thèse de Doctorat, Université Paul Sabatier, Toulouse.

Sakai, S. (2001) Phenological diversity in tropical forests. Population Ecology, 43, 77-86

Schupp, E.W. (1988). Factors affecting postdispersal seed survival in a tropical forest. Oecologia, 76, 525-530.
Sollins, P. (1998). Factors influencing species composition in tropical lowland rain forest: Does soil matter? Tropical montane forests. Ecology, 79, 23-30.

Stevenson, P. (2004). Fruit choice by wolly monkeys in Tinigua national Park, Colombia. International Journal of Primatology, 25, 367-381.

Swaine, M.D. (1996). Rainfall and soil fertility as factors limiting forests species distributions in Ghana. Journal of Ecology, 84, 419-428

Ter Steege, H., Pitman, N.C.A., Phillips, O.L., Chave, J., Sabatier, D., Duque, A., Molino J.F., Prévost, M.F., Spichiger, R., Castellanos, H., von Hildebrand, P. \& Vásquez, R. (2006). Continental-scale patterns of canopy tree composition and function across Amazonia. Nature, 443, 444-447.

Ter Steege et al. (2013). Hyperdominance in the Amazonian tree flora. Science, 342, 1243092

Terborgh, J. (2012). Enemies maintain hyperdiverse tropical forests. American Naturalist, 179, 303-314.

Tilman, D. (1994). Competition and biodiversity in spatially structured habitats. Ecology, 75, 2-16.

Tilman, D. (1999) Diversity by default. Science, 283, 495-496.

Turnbull, L.A., Crawley, M.J. \& Rees, M. (2000) Are plant populations seed-limited? A review of seed sowing experiments. Oikos, $88,225-238$.

Uriarte M, Schneider L \& Rudel TK. (2009). Synthesis: Land Transitions in the Tropics. Biotropica, 42, 59-62. 
Valencia, R., Foster, R.B., Villa G., Condit R., Svenning, J.-C., Hernández, C., Romoleroux, K., Losos, E., Magard, E. \& Balslev, H. (2004) Tree species distributions and local habitat variation in the Amazon: large forest plot in eastern Ecuador. Journal of Ecology, 92, 214-229.

Vargas-Rodríguez, Y.L., Váquez-García, J.A. \& Williamson, B. (2005). Environmental correlates of tree and seedling-sapling distribution in a Mexican tropical dry. Plant Ecology, 180, 117-134

Wang, B.C. \& Smith, T.B. (2002) Closing the seed dispersal loop. Trends in Ecology and Evolution, 17, 379-385

Warner, R.R. \& Chesson, P.L. (1985) Coexistence mediated by recruitment fluctuations: a field guide to the storage effect. American Naturalist, 125, 769-787.

Webb, C.O. \& Peart, D.R. (2000) Habitat associations of trees and seedlings in a Bornean rain forest. Journal of Ecology, $88,464-478$.
Whittaker, R.H. (1956) Vegetation of the Great Smoky Mountains. Ecological Monographs, 26, 1-80.

Wright, S.J. \& Calderón, O. (1995) Phylogenetic patterns among tropical flowering phenologies. Journal of Ecology, 83, 937948.

Wright, S.J., Carrasco, C., Calderón, O. \& Patón, S. (1999) The El Nino Southern Oscillation variable fruit production, and famine in a tropical forest. Ecology, 80, 1632-1647.

Wright, S.J., Muller-Landau, H.C., Calderón, O. \& Hernández, A. (2005) Annual and spatial variation in seedfall and seedling recruitment in a neotropical forest. Ecology, 86, 848-860.

Young, T.P., Petersen, D.A., \& Clary, J.J. (2005). The ecology of restoration : historical links, emerging issues and unexplored realms. Ecology Letters, 8, 662-673. 